\title{
Thrombospondin 1 Modulates Monocyte Properties to Suppress Intestinal Mucosal Inflammation
}

\author{
Lei-Lei Fang $^{a}$ Hai-Qiong Yu ${ }^{b, c}$ Rui-Jin Wu ${ }^{a}$ Chong He ${ }^{a}$ Meng Lib,c Hao Yan ${ }^{b, c}$ \\ Jian-Jie Lib,c Shuai Wang ${ }^{\text {b, c }}$ Zhi-Gang Liub,c Zhan-Ju Liu ${ }^{a}$ Ping-Chang Yang ${ }^{\text {b, c }}$ \\ aDepartment of Gastroenterology, Shanghai Tenth People's Hospital of Tongji University, Shanghai, and 'bShenzhen \\ Key Laboratory of Allergy and Immunology, ENT Institute, Shenzhen University School of Medicine, and 'State Key \\ Laboratory of Respiratory Disease for Allergy at Shenzhen University, Shenzhen, China
}

\section{Key Words}

Monocytes · Intestinal inflammation · Probiotics .

Regulatory T cells $\cdot$ Mucosal immunology

\begin{abstract}
Monocytes (Mos) play an important role in the pathogenesis of intestinal mucosal inflammation. This study aims to investigate the mechanism by which the intestinal epithelial cellderived thrombospondin 1 (TSP1) modulates Mo properties and regulates intestinal inflammatory responses. In this study, the production of TSP1 by intestinal epithelial cells was evaluated by quantitative real-time PCR and Western blotting. The properties of Mos were analyzed by flow cytometry. A mouse model of colitis was created to assess the role of epithelium-derived TSP1 in the suppression of intestinal inflammation. The results demonstrated that mouse intestinal epithelial cells (IECs) expressed TSP1, which was markedly upregulated by butyrate or feeding with Clostridium butyricum. Coculture of the butyrate-primed IECs and Mos or exposure of Mos to TSP1 in the culture induced the expression of transforming growth factor (TGF)- $\beta$ in Mos.
\end{abstract}

These TGF- $\beta^{+}$Mos had tolerogenic properties that could promote generation of inducible regulatory $T$ cells. Adoptive transfer with TSP1-primed Mos, or feeding C. butyricum could prevent experimental colitis in mice. In summary, $C$. butyricum induces intestinal epithelial cells to produce TSP1 and induces TGF- $\beta^{+}$Mos, which further suppress experimental colitis in mice. The results implicate that the administration of $C$. butyricum or butyrate may have the potential to ameliorate chronic intestinal inflammation through inducing immunosuppressive Mos.

(c) 2015 S. Karger AG, Basel

\section{Introduction}

Inflammatory bowel disease (IBD), including Crohn's disease and ulcerative colitis, is characterized by chronic inflammation in the intestinal tract. The pathogenesis is unclear. It is thought to be driven by the immune system that overreacts to microbial and environmental factors in genetically predisposed individuals [1]. Many immune cells and inflammatory mediators, such as T cells, macro-

\section{L.-L.F. and H.-Q.Y. share the first authorship.}

(c) 2015 S. Karger AG, Basel

$1662-811 X / 15 / 0076-0601 \$ 39.50 / 0$
Dr. Zhan-Ju Liu

Department of Gastroenterology, Shanghai Tenth People's Hospital of Tongji University Shanghai 200072 (China)

E-Mail liuzhanju88@126.com

Dr. Ping-Chang Yang

State Key Laboratory of Respiratory Disease for Allergy at Shenzhen University Shenzhen 518060 (China)

E-Mail pcy2356@163.com 
phages and cytokines, have been implicated as playing roles in the development of IBD. T helper (Th)1 polarization is generally considered to play a major role in the pathogenesis of Crohn's disease, while Th2 polarization seems to be involved in the development of ulcerative colitis. Moreover, Th17 polarization has also been found involved in mucosal inflammation in IBD [2]. Published data have demonstrated that regulatory $\mathrm{T}\left(\mathrm{T}_{\text {reg }}\right)$ cells play an essential role in suppressing skewed immune responses [3]. It is known that transforming growth factor (TGF) $\beta$ is one of the most important mediators for the suppressive functions of $\mathrm{T}_{\text {reg }}$ cells. However, $\mathrm{CD} 4^{+}$ $\mathrm{CD} 25^{\text {low }}$ Foxp $3^{+} \mathrm{T}$ cells which lose the Foxp3 expression are prone to differentiate into Th17 cells in some immune diseases such as arthritis [4] and IBD. However, the mechanisms involved in the regulation of $\mathrm{T}_{\text {reg }}$ cell differentiation and function are not fully understood.

It is suggested that monocytes (Mos) may play a selective role in initiating the differentiation of immunosuppressive cells [5]. The intestine contains the largest pool of Mos in the body. Mos consist of 2-10\% of all leukocytes in the human body. After activation, Mos migrate from the bloodstream to other tissues to differentiate into tissue-resident macrophages or dendritic cells. It is reported that a subfraction of Mos plays a key role in the immuneregulatory activities, such as the myeloid-derived suppressor cells which function as immunosuppressive cells and block both innate and adaptive antitumor immunity [6]. It is also suggested that expansion of myeloid-derived suppressor cells suppresses IBD-like inflammation in animal models [7], which sheds a new light on the treatment of IBD. However, how to generate the Mos with immunosuppressive functions is unclear.

Thrombospondins (TSPs) are originally reported as a component of platelet $\alpha$-granules [8]. TSP1, the first natural protein angiogenesis inhibitor identified by Good et al. [9], is best studied in this family. Besides platelets, it is also expressed by endothelial cells, adipocytes, fibroblasts, smooth muscle cells, Mos and transformed cells, such as malignant glioma cells, dendritic cells and B cells [10]. It interacts with CD36, a fatty acid translocase receptor on the endothelial cell membrane, and various types of cells [11]. As mentioned above, CD36 also presents in Mos to act as scavenger receptor. However, whether the TSP1mediated signal is involved not only in antiangiogenesis, but also in Mo immune responses is largely unclear yet.

Clostridium butyricum, a strictly anaerobic bacillus, produces butyric acid. It has been safely used as a therapeutic supplement for severely ill, immunocompromised and hospitalized subjects from infants to elderly people, including pregnant women [12]. The amelioration of intestinal inflammation by probiotics has been recognized [13], but the underlying mechanism remains to be further investigated. Our previous study has revealed that TSP1 can convert the latent TGF- $\beta$ to the active TGF- $\beta$, suggesting that TSP1 plays an important role in the generation of immune tolerance via inducing $\mathrm{T}_{\text {reg }}$ cells [10]. Therefore, we hypothesize that TSP1 facilitates Mos to produce TGF- $\beta$ and then drives naïve $\mathrm{CD} 4^{+} \mathrm{T}$ cells to differentiate into $\mathrm{T}_{\text {reg }}$ cells in the gut mucosa to suppress the mucosal inflammation. In the present study, we observed that $C$. butyricum induced intestinal epithelial cells (IECs) to produce TSP 1 . This IEC-derived TSP 1 facilitated naïve Mos to differentiate into Mos producing high levels of TGF- $\beta$ and further significantly inhibited experimental colitis in mice, which implicates that the Mos with immunosuppressive functions have therapeutic potential in the treatment of IBD.

\section{Materials and Methods}

\section{Reagents}

Fluorescence-labeled antibodies of TGF- $\beta$, CD36, CD80, CD64, F4/80, tumor necrosis factor (TNF)- $\alpha$, NK1.1, CD3, CD14, CD19, CD20 and interferon (IFN) $-\gamma$ were purchased from BD Bioscience (BD Bioscience, San Diego, Calif., USA). The small hairpin RNA (shRNA) kits of CD36, histone deacetylase (HDAC) 1 and HDAC2, antibodies of CD36, HDAC1, HDAC2 and TSP1 were purchased from Santa Cruz Biotech (Dallas, Tex., USA). TSP1, antagonist peptide LSKL and SLLK (Ser-Leu-Leu-Lys) were synthesized by TradeTT (Beijing, China). Reagents for quantitative realtime polymerase chain reaction (qRT-PCR) and Western blotting were purchased from Invitrogen (Carlsbad, Calif., USA). Magnetic bead-conjugated streptavidin was purchased from Miltenyi Biotech (Germany).

\section{Mice}

Male C57BL/6 mice (8-12 weeks old) were purchased from the Guangdong Experimental Animal Center (Guangdong, China). Reg $2^{-l-}$ mice were purchased from the Xinmao Experimental Animal Institute (Shanghai, China). B cell-deficient mice were generated by intraperitoneal injection with anti-CD20 monoclonal antibody at $0.25 \mathrm{mg} / \mathrm{mouse}$ ( 1 week later, no B cells were found in the intestine, the spleen and the peripheral blood as assessed by flow cytometry; data not shown). Mice were kept in a pathogen-free environment at Shenzhen University (Shenzhen, China) during the experimental period. The experimental procedures were approved by the Experimental Animal Ethic Committee at Shenzhen University.

\section{IEC Culture}

The mouse intestinal epithelial cell line IEC-4.1 was cultured in Dulbecco's modification of Eagle's medium supplemented with $10 \%$ fetal bovine serum, $2 \mathrm{mM}$ L-glutamine, $100 \mathrm{U} / \mathrm{ml}$ penicillin and $100 \mathrm{mg} / \mathrm{ml}$ streptomycin. The medium was changed every $1-2$ days. A Transwell system was employed for a coculture experiment 
of IECs and Mos: IECs were seeded in the inserts while Mos were placed in the basal chambers. The cell viability was assessed by the trypan blue exclusion assay.

\section{Quantitative Real-Time PCR}

Total RNA was extracted from IECs using the Trizol reagents following a protocol obtained from the manufacturer. The quantity and quality were assessed using a NanoVue spectrophotometer, with a $260 / 280$ ratio of $>1.8$ and $28 \mathrm{~S} / 18 \mathrm{~S}$ ratio of $>1.4$ for the majority of the samples. cDNA was synthesized from $2 \mu \mathrm{g}$ of total RNA using a reverse conversion kit. PCR amplification was performed on a MiniOpticon ${ }^{\mathrm{TM}}$ Real-Time PCR Detection System using a SYBR ${ }^{\circledR}$ Green PCR kit. The differences in target gene expression were expressed relatively to the $\beta$-actin gene using the $2^{-\Delta \Delta \mathrm{C}_{\mathrm{T}}}$ method $\left(\Delta \Delta \mathrm{C}_{\mathrm{T}}=\right.$ average of $\Delta \mathrm{C}_{\mathrm{T}}$ control $-\Delta \mathrm{C}_{\mathrm{T}}$ treated $)$. The primers used in this study include: TSP1, forward, caactcccagctgtgcaatt; reverse, gccgaggtgaacacaaaact; TGF- $\beta$, forward, ggtgagaagctgaagaccct; reverse, tgtctaggtcctggagtcca, IFN- $\gamma$, forward, ttcttcagcaacagcaaggc; reverse, actcctttccgcttcctga; TNF- $\alpha$, forward, gaccctttactctgacccc; reverse, aggctccagtgaattcg gaa.

\section{Western Blotting}

Total protein was extracted from the cells, fractioned in sodium dodecyl sulfate polyacrylamide gel electrophoresis and transferred onto a nitrocellulose membrane. After blocking with 5\% skimmed milk for $30 \mathrm{~min}$, the membrane was incubated with the primary antibodies $(0.3-0.5 \mu \mathrm{g} / \mathrm{ml})$ at room temperature for $1 \mathrm{~h}$ and followed by the second antibody conjugated with horseradish peroxidase $(0.05-0.1 \mu \mathrm{g} / \mathrm{ml})$ at room temperature for $1 \mathrm{~h}$. Washing with Tris-buffered saline with Tween-20 was performed after each antibody incubation. The immune complex on the membrane was developed with enhanced chemiluminescence. The results were photographed using the Kodak Imaging Station 4,000 mm Pro (Shanghai, China). The integrated density of the immune blots was assessed with software of Photoshop (CS5).

\section{Immunohistochemistry}

Mouse colon segments were frozen in liquid nitrogen. Cryosections were prepared, fixed with cold acetone and blocked with $1 \%$ bovine serum albumin. The sections were incubated with the first antibodies $(1 \mu \mathrm{g} / \mathrm{ml})$ for $1 \mathrm{~h}$ at room temperature, followed by incubating with the secondary antibodies $(1 \mathrm{~h})$, and then incubated with the fluorescein-labeled antibodies against the hosts of the secondary antibodies $(1 \mathrm{~h})$. After washing with PBS, the sections were finally stained with propidium iodide $(5 \mu \mathrm{g} / \mathrm{ml})$ for $10 \mathrm{~min}$ to stain the nucleus, mounted with cover slips, and observed with a confocal microscope (Zeiss LSM510, Germany). The observers were not aware of the code to avoid observer bias.

\section{Isolation of Immune Cells from the Intestinal Segments}

Lamina propria mononuclear cells (LPMCs) were isolated as previously reported [14]. Briefly, colons and small intestines were opened longitudinally and rinsed in PBS. Intestines were incubated while mildly shaken in Hanks' balanced salt solution containing $5 \mathrm{mM}$ EDTA and $5 \%$ fetal bovine serum for $30 \mathrm{~min}$ at $37^{\circ} \mathrm{C}$. After removal of epithelial layers, the intestines were cut into $2 \times 2 \times$ $2 \mathrm{~mm}$ pieces and incubated with RPMI 1640 containing 5\% fetal bovine serum, $1 \mathrm{mg} / \mathrm{ml}$ of collagenase IV (Roche, Mannheim,
Germany) for $30 \mathrm{~min}$ at $37^{\circ} \mathrm{C}$ while mildly shaken. The digested tissues were washed with Hanks's balanced salt solution containing $5 \mathrm{mM}$ EDTA. Cell suspensions were filtered through a $70-\mu \mathrm{m}$ cell strainer. The cells were collected and further purified via density gradient centrifugation with 40 and 70\% Percoll-RPMI solution [15]. The cells were resuspended and were applied to gradient centrifugation at $1,000 \mathrm{~g}$ for $40 \mathrm{~min}$. The viability was greater than $98 \%$ as determined by the trypan blue exclusion assay. The individual immune cell type was further isolated by magnetic cell sorting (MACS) with commercial reagent kits following the manufacturer's instructions.

\section{Flow Cytometry}

Cells were fixed with $2 \%$ paraformaldehyde (in the case of the intracellular staining, $0.1 \%$ Triton $\mathrm{X}-100$ was added to the fixative to enhance the permeation of the cell membrane) for $30 \mathrm{~min}$, washed with PBS 3 times and incubated with the fluorescence-labeled antibodies $(0.5-1 \mu \mathrm{g} / \mathrm{ml})$ for $30 \mathrm{~min}$ at room temperature. For isotype control staining, the matched antibody isotype (or fluorescence minus one) [16] was used at the same concentration as the antibody. After washing with PBS, the cells were analyzed by flow cytometry (FACSCanto II, BD, USA). 100,000 cells were counted for each sample. The data were analyzed with the software Flowjo (Tree star, Ashland, Oreg., USA). The gates were set using the isotype staining results as a guide.

\section{Preparation of Conditioned Mos}

$\mathrm{CD} 14^{+} \mathrm{CD} 16^{-} \mathrm{MHC} \mathrm{II}^{-}$Mos were isolated from the bone marrow by MACS and cultured with IECs at a ratio of 1:1 in the Transwell system in the presence of recombinant butyrate $(2.5 \mathrm{mM})$ for $72 \mathrm{~h}$ (the two cell types were separately cultured). The conditioned Mos were collected from the basal chambers and used for further experiments. On the other hand, Mos were also isolated by MACS from the LPMCs of both naive mice and the mice treated with C. butyricum.

\section{Induction of Colitis in Mice}

C57BL/6 mice drank water containing dextran sulfate sodium (DSS; $5 \%$; w/v) for 8 days; control mice drank water instead. The mice were monitored daily for weight, morbidity and stool consistency, and sacrificed on day 12 . The length of the colon was measured. A piece of colon was excised to test the myeloperoxidase (MPO) activity. Another piece of colon was processed for hematoxylin and eosin staining, and histological scores were determined.

\section{Treatment of Colitic Mice with Mos}

On day 0 , DSS-induced colitis mice were injected with TSP1conditioned Mos or naïve Mos $\left(1 \times 10^{6}\right.$ cells/mouse $)$ via the tail vein, gavage feeding with $0.3 \mathrm{ml} \mathrm{C.} \mathrm{butyricum}\left(10^{9}\right.$ cells $\left./ \mathrm{ml}\right)$ or injected (intraperitoneally) with sodium butyrate $(1.2 \mathrm{mg} / \mathrm{kg}$ body weight). The treatment was repeated on day 3 .

\section{Administration of TSP1 Inhibitors}

Peptides LSKL and SLLK were dissolved in saline $(1.0 \mathrm{mg} / \mathrm{ml})$, and injected intraperitoneally at $30 \mathrm{mg} / \mathrm{kg}$ body weight.

\section{Histological Scoring of Colitis}

After sacrifice, the transverse colons were removed from the mice, fixed with $4 \%$ paraformaldehyde and embedded in paraffin. The tissue sections were stained with hematoxylin-eosin or peri- 
odic acid-Schiff stain. The inflammation in intestinal sections was scored following the published criteria [15]. Histological inflammatory scores were recorded as follows: (a) the severity of inflammation: 0 , none; 1 , mild lymphoid infiltration; 2 , marked lymphoid infiltration or focal degeneration of crypts; 3 , severe inflammation or multifocal crypt degeneration and/or erosions; (b) the extent of inflammation: 0 , none; 1 , mucosal; 2 , submucosal; 3 , transmural; (c) the amount of mucus: 0 , normal; 1, slight decrease in mucus; 2 , moderate decrease or focal absence of mucus; 3 , severe depletion of mucus; 4 , total absence of mucus; (d) the degree of cell proliferation: 0 , none; 1 , mild increase in cell numbers and crypt length; 2 , moderate increase or focally marked increase; 3 , marked increase in the entire section. The inflammatory scores were calculated according to the sum of the four individual parameters.

\section{RNA Interference}

CD36, HDAC1 and HDAC2 were knocked down in the cells by RNA interference (RNAi) with shRNA reagent kits according to the manufacturer's instruction. The effect of RNAi was determined by Western blotting.

\section{T-Cell Proliferation Assay}

$\mathrm{CD}^{+} \mathrm{CD}^{+} \mathrm{CD}^{-} 5^{-} \mathrm{T}$ cells ( $\mathrm{T}_{\text {eff }}$ cells) were isolated from the mouse spleen and labeled with carboxyfluorescein diacetate succinimidyl ester (CFSE). The $\mathrm{T}_{\text {eff }}$ cells were cultured in a plate coated with anti-CD3 antibody $(5 \mu \mathrm{g} / \mathrm{ml})$, and anti-CD28 antibody $(2 \mu \mathrm{g} / \mathrm{ml})$ was added to the culture. Three days later, the cells were analyzed by flow cytometry.

\section{Measurement of MPO Activity}

The MPO activity assay was performed with the colon tissues according to the manufacturer's instructions of the MPO assay kit (Biomart, Shenzhen, China). Colon segments were homogenized at $50 \mathrm{mg} / \mathrm{ml}$ in phosphate buffer $(50 \mathrm{mmol} / \mathrm{l}, \mathrm{pH} 6.0)$ with $0.5 \%$ hexadecyltrimethylammonium bromide. The homogenates were frozen-thawed 3 times, centrifuged at $40,000 \mathrm{~g}$, and then $0.1-\mathrm{ml}$ aliquots were mixed with $2.9 \mathrm{ml}$ phosphate buffer $(\mathrm{pH}=6.0)$ containing $0.167 \mathrm{mg} / \mathrm{ml} o$-dianisidine and $5 \times 10^{-4} \mathrm{H}_{2} \mathrm{O}_{2}$ and incubated at $25^{\circ} \mathrm{C}$ for $20 \mathrm{~min}$. The optical density was measured at $460 \mathrm{~nm}$. The MPO activity was expressed in units per gram of tissue ( 1 unit corresponded to the activity required to degrade $1 \mathrm{mM}$ of hydrogen peroxide per minute at $25^{\circ} \mathrm{C}$ ).

\section{Statistical Analysis}

Data are expressed as means \pm SD. Differences between 2 groups were determined by Student's $t$ test. In the case of more than 2 groups, 1-way ANOVA was employed. When the results of ANOVA were significant, the Tukey-Kramer honestly significant difference test was applied for multiple comparisons. $\mathrm{p}<0.05$ was set as a criterion of significance.

\section{Results}

\section{IECs Produce TSP1}

Our previous study revealed that B cell-derived TSP1 played a role in the suppression of intestinal inflammation [10]. Whether TSP1 also comes from the intestinal components is unclear. To this end, we assessed the expression of TSP1 in IECs. The results showed that TSP1 was detectable in naïve IECs, which was markedly increased when IECs were activated by phorbol myristate acetate in the culture (fig. 1a, c). The expression of TSP1 was also detected in the naïve mouse colon epithelia, which was upregulated by feeding mice with C. butyricum daily for 5 days (fig. $1 \mathrm{~b}, \mathrm{~d}-\mathrm{g}$ ). Since one of the molecules produced by C. butyricum is butyrate, we then injected mice with sodium butyrate, or added sodium butyrate to IEC cultures. Interestingly, C. butyricum was found to promote the expression of TSP 1 in the colon epithelia and IECs (fig. 1b, d). Since a fraction of B cells also express TSP1 [10], we isolated LPMCs from the colon of mice treated with saline or C. butyricum and analyzed them by flow cytometry. The results showed that a small fraction of TSP $1^{+}$dendritic cells and TSP $1^{+} \mathrm{B}$ cells was detected in the LPMCs, which was not upregulated by the administration with C. butyricum (fig. $1 \mathrm{~h}-\mathrm{m}$ ). The results indicate that mouse epithelial cells express TSP1, which can be upregulated by phorbol myristate acetate and C. butyricum-derived butyrate.

\section{HDAC1 Restricts the Expression of TSP1 in IEC}

Since butyrate is an inhibitor of HDAC1 and HDAC2, the data from figure 1 indicate that butyrate could promote the expression of TSP1 in IECs. We wondered whether HDAC1 or/and HDAC2 could restrict the expression of TSP 1 in IECs. To test the hypothesis, the genes of HDAC1 (fig. 2a) or HDAC2 (fig. 2b) were silenced in IECs, or overexpressed HDAC1 in IECs (fig. 2c). As shown by qRT-PCR and Western blotting, the gene silencing HDAC1, but not HDAC2, significantly enhanced the levels of TSP1 in IECs (fig. 2d, e). The addition of butyrate to the culture did not further increase the expression of TSP1 (fig. 2d, e). Overexpression of HDAC1 further suppressed the expression of TSP1 in IECs (fig. $2 \mathrm{~d}$, e). The results suggest that $\mathrm{HDAC} 1$ is a negative regulator of the expression of TSP1 in IECs.

\section{CD $36^{+}$Mos Express TGF- $\beta$}

Our previous data show that the B cell-derived TSP1 has immune-regulatory properties in the intestine [10]. We inferred that Mos might be influenced by TSP 1 . Thus, LPMCs were isolated from the naïve mouse colon. The Mos were gated from the LPMCs with flow cytometry (fig. 3a). The gated Mos showed 25.4\% Mos from the naïve mouse colon expressed CD36, a TSP1 receptor (fig. 3b). After activation, Mos may further differentiate into macrophages. Thus, we further analyzed some of the 


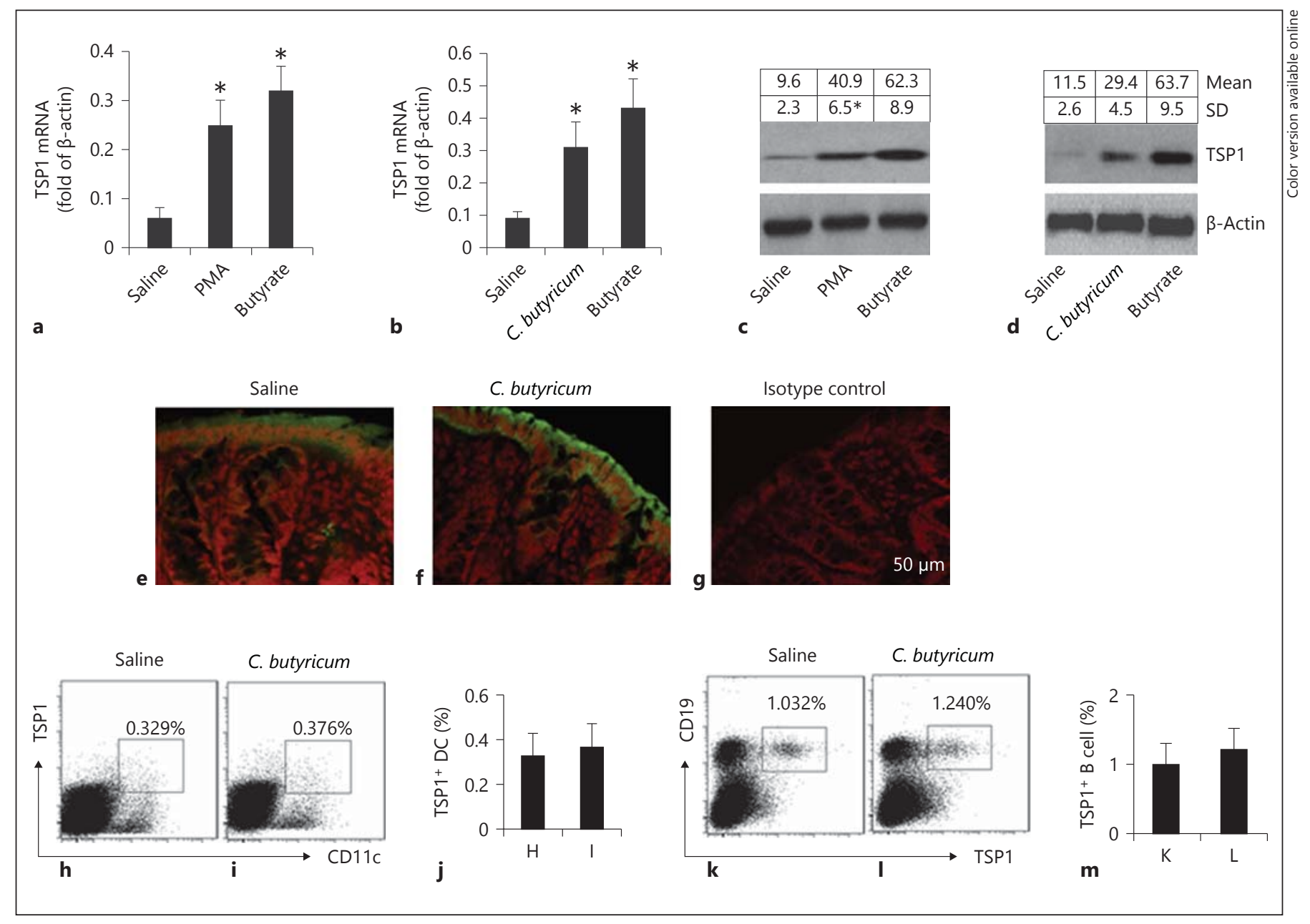

Fig. 1. Intestinal epithelial cells express TSP1. A mouse small intestinal epithelial cell line, IECs, was cultured. The treatment is denoted on the $\mathrm{x}$-axis of $\mathbf{a}$ and $\mathbf{b}$, and above each image. Total RNA and proteins were extracted from IECs or the mouse colon epithelia and analyzed by qRT-PCR and Western blotting. $\mathbf{a}, \mathbf{b}$ The bars indicate the mRNA levels of TSP1 in IECs (a) and the colon epithelia (b). PMA = Phorbol myristate acetate $(10 \mathrm{ng} / \mathrm{ml})$. c, $\mathbf{d}$ The immune blots indicate the protein levels of TSP1 in IECs (c) and the colon epithelia (d). The table shows the integrated density of the immune blots. e-I C57BL/6 mice were treated with saline or C. butyricum daily for 5 days. e-g The confocal images indicate TSP1 staining (in green; in the online version only) in the colon epithelia. Magnifica- tion of the images: $\times 200$. $\mathbf{h}-\mathbf{I}$ The LPMCs were isolated from the mouse colon and analyzed by flow cytometry. $\mathbf{h}$, i The gated dot plots indicate the frequency of TSP $1^{+}$dendritic cells (DCs). $\mathbf{j}$ The bars indicate the summarized data of $\mathbf{h}$ and $\mathbf{i} . \mathbf{k}$, I The gated dot plots indicate the frequency of TSP $1^{+} \mathrm{B}$ cells. $\mathbf{m}$ The bars indicate the summarized data of $\mathbf{k}$ and $\mathbf{I}$. C. butyricum: each mouse was fed with $0.3 \mathrm{ml}$ of $10^{9}$ C. butyricum $/ \mathrm{ml}$ daily; butyrate: sodium butyrate was administered to mice as $1.2 \mathrm{~g} / \mathrm{kg}$ once-daily intraperitoneal injections for 5 days. The data of bars are presented as means \pm SD. ${ }^{*} \mathrm{p}<$ 0.01 , compared with the saline group. The data are representative of 3 independent cell culture experiments. Each group consists of 6 mice. Samples from individual mice were processed separately. cytokines of macrophages by flow cytometry. The results revealed that $\mathrm{CD}^{2} 6^{+}$Mos were also TGF- $\beta^{+}$TNF- $\alpha^{-}$ $\mathrm{CD} 80^{\text {low }} \mathrm{CD}^{-} 4^{-} \mathrm{F} 4 / 80^{-}$(fig. 3c-g) while the $\mathrm{CD} 36^{-}$Mos were TGF- $\beta^{-}$TNF- $\alpha^{+}$CD $80^{\text {high }}$ CD $64^{-}$F $4 / 80^{\text {low }}$ (fig. 3h1). In addition, we also phenotyped the $\mathrm{CD}^{+} 6^{+}$cells in LPMCs by flow cytometry, which was NK1.1 $1^{+}(16.6 \%)$, $\mathrm{CD}^{+}(20.7 \%), \mathrm{CD}^{+} 4^{+}(31.9 \%)$ and $\mathrm{CD}^{+} 9^{+}(19.5 \%)$, respectively (fig. $3 \mathrm{~m}-\mathrm{q})$.

\section{C. butyricum Modulates Mos Tolerogenic Property via Epithelial Cell-Derived TSP1}

Mos distribute in the subepithelial region of the intestine. Such an anatomical feature implicates that the epithelial cell-derived TSP1 may have the potential to regulate the properties and functions of Mos. To this end, we treated naïve $\mathrm{C} 57 \mathrm{BL} / 6$ mice as denoted in figure 4 . The flow cytometry data showed that feeding with C. butyricum 
Fig. 2. HDAC1 restricts the expression of TSP1 in IECs. IECs were treated with shRNA of HDAC1 (a) or shRNA of HDAC2 (b), or overexpressed HDAC1 (c). The IEC extracts were analyzed to determine the levels of TSP1. $\mathbf{d}$ The bars indicate the mRNA levels of TSP1. e The immune blots indicate the protein contents of TSP1. The bars indicate the integrated density of the immune blots. HDAC1- $\mathrm{n}=$ HDAC1null IECs; HDAC2-n = HDAC2-null IECs; cshRNA = IECs were treated with control shRNA; HDAC1-n-b = HDAC1-null IECs were treated with butyrate in the culture; HDAC1-Oex = IECs were overexpressed by HDAC1; E-plasmid = IECs were treated with an empty plasmid used as a control.

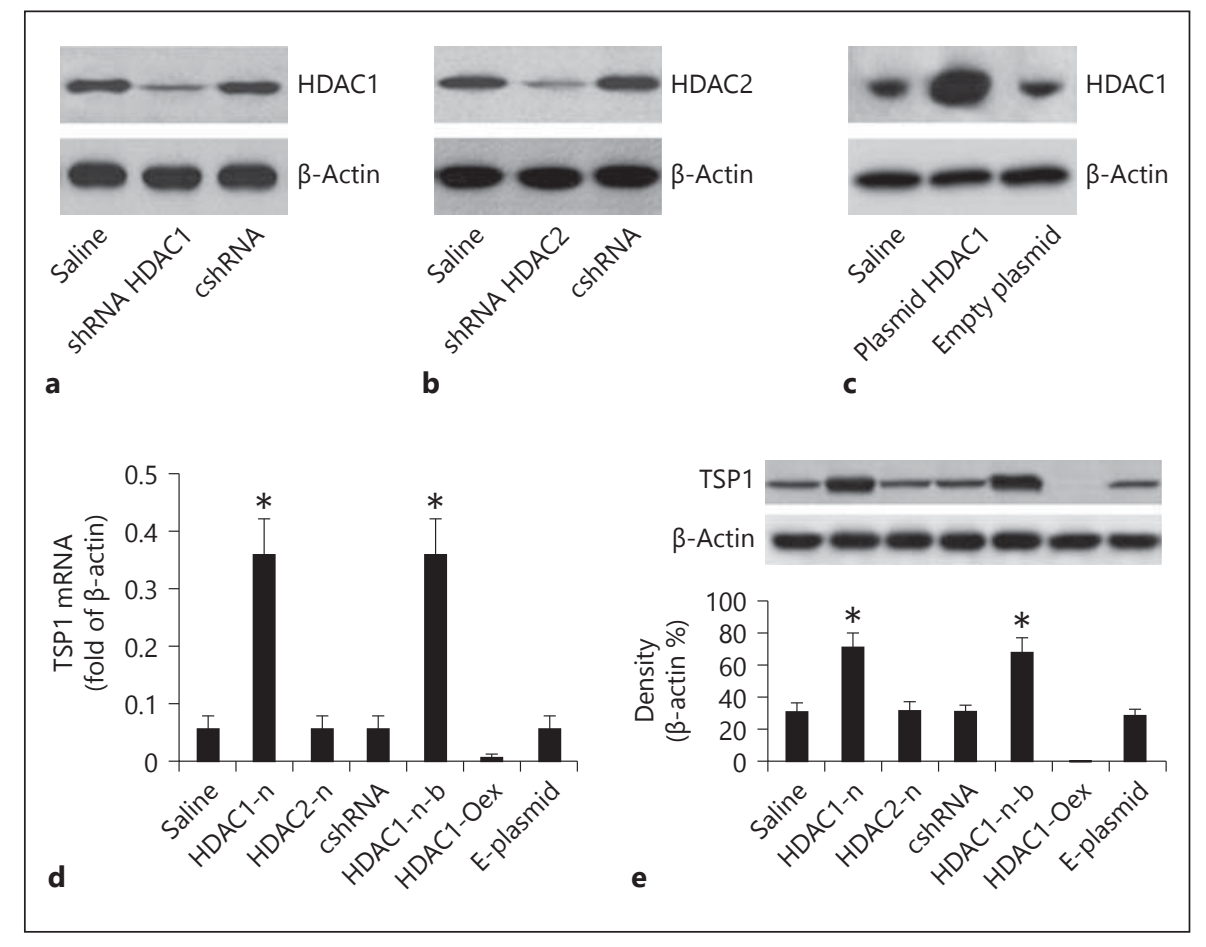

markedly increased the frequency of TGF- $\beta^{+}$Mos in the gut (fig. 4a-d). To test whether TSP1 was a specific molecule to mediate the effect of $C$. butyricum on inducing the TGF- $\beta^{+}$Mos, we treated mice with a TSP1 inhibitor (SLLK) while feeding with C. butyricum. As expected, the induction of TGF- $\beta^{+}$Mos was abolished (fig. $4 \mathrm{e}-\mathrm{h}$ ). To elucidate if T cells or B cells were required in the C. butyricum-induced TGF- $\beta^{-}$Mos in the intestine, we treated T cell-deficient Reg2 ${ }^{-/}$mice or B cell-deficient mice with $C$. butyricum. The TGF- $\beta^{+}$Mos were still increased in the intestine (fig. $4 \mathrm{i}-1$ ). The results suggest that the C. butyricum-induced TGF- $\beta^{-}$Mos in the intestine are not T cellor B cell-dependent.

To strengthen the data of figure $4 a-1$, naïve Mos were treated with TSP1 in the culture for $72 \mathrm{~h}$. After exposure to TSP1 in the culture, the expression of TGF- $\beta$ was markedly upregulated in a TSP1 dose-dependent manner, which was abolished in the CD36-deficient Mos. Coculture of naive Mos and IECs in the presence of sodium butyrate also induced TGF- $\beta$ in Mos, but not in CD36deficient Mos. However, exposing naïve Mos to sodium butyrate in the absence of IECs could not induce TGF- $\beta$ expression in Mos (fig. $4 \mathrm{~m}, \mathrm{n}$ ). This result was further supported by the additional data, in which TSP 1 induced the expression of TGF- $\beta$ in Mos, but not in those treated with saline or lipopolysaccharide (fig. 4o-r). The data suggest that the C. butyricum-derived butyrate induces IECs to produce TSP1, which in turn induces expression of TGF- $\beta$ in Mos.

\section{TSP1-Primed Mos Show Tolerogenic Features}

To test whether the TSP1-primed Mos have tolerogenic features, we next treated naïve Mos with TSP1 (200 $\mathrm{ng} / \mathrm{ml}$ ) in the culture for $72 \mathrm{~h}$. The TSP1-primed Mos were cultured with $\mathrm{T}_{\text {eff }}$ cells $\left(\mathrm{CD}^{+} \mathrm{CD}^{+} \mathrm{CD} 25^{-} \mathrm{T}\right.$ cells) at a ratio of 1:1 for 6 days. The results showed that the TSP1-primed Mos induced the $\mathrm{T}_{\text {eff }}$ cells to differentiate into TGF- $\beta^{+} \mathrm{T}_{\text {reg }}$ cells (fig. $5 \mathrm{a}$, e). The culture with naïve Mos did not induce apparent $\mathrm{T}_{\text {reg }}$ development (fig. 5b, e). Since the expression of CD36 was much higher on Mos than on T cells, we cultured naïve Mos and $\mathrm{T}_{\text {eff }}$ cells, TSP 1 was added to the culture, and the cells were cultured for 6 days. As analyzed by flow cytometry, T regulation was induced (fig. $5 c$, e). Treating $\mathrm{T}$ cells with TSP1 without the presence of Mos did not induce detectable $\mathrm{T}_{\text {reg }}$ cells (fig. $5 \mathrm{~d}, \mathrm{e}$ ). To strengthen the results, we isolated intestinal Mos from mice treated with C. butyricum for 5 days; the Mos were cultured with naïve $\mathrm{CD} 4^{+}$ T cells for 6 days. The cells were analyzed by flow cytometry. The results showed that the Mos from C. butyricumtreated mice markedly increased the naïve $\mathrm{CD} 4^{+} \mathrm{T}$ cells to $\mathrm{T}_{\text {reg }}$ cells (fig. $5 \mathrm{f}$ ), which did not occur in those $\mathrm{CD} 4^{+}$ 


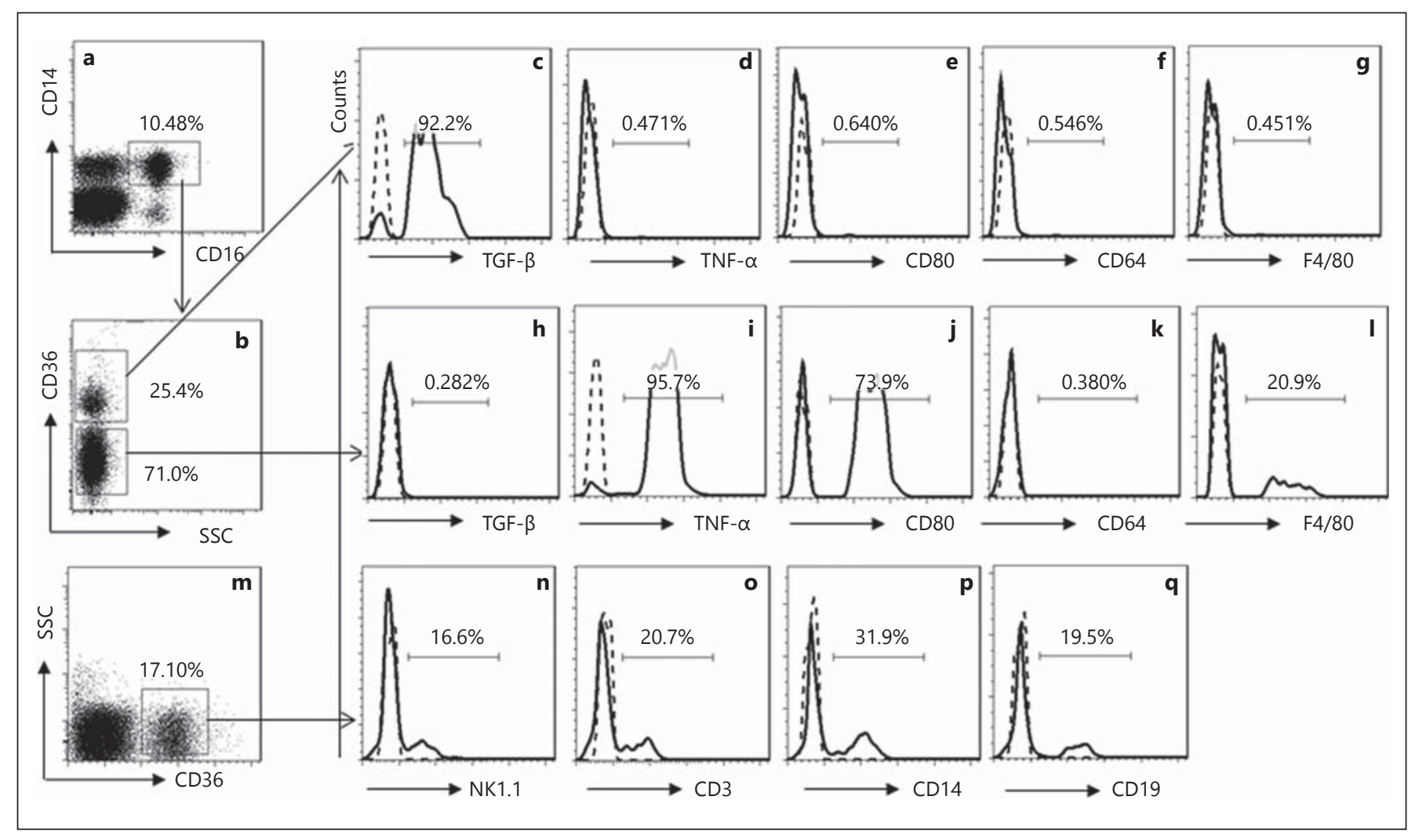

Fig. 3. Phenotypes of $\mathrm{CD} 36^{+}$and $\mathrm{CD}^{-} 6^{-}$Mos. LPMCs were isolated from the naïve C57BL/6 mouse intestine, stained with the indicated antibodies (denoted in each subpanel) and analyzed by flow cytometry. SSC = Side scatter. a The dot plots indicate the frequency of Mos in LPMCs. b The dot plots indicate the CD36 ${ }^{+}$ and CD $36^{-}$Mos in the gated Mos of a. c-I The histograms indicate the phenotypes (denoted below each histogram) of $\mathrm{CD}^{+} 6^{+} \mathrm{Mos}$ (c-g) and $\mathrm{CD}^{-} 6^{-}$Mos (h-I). $\mathbf{m}$ The gated dot plots show the total frequency of $\mathrm{CD} 36^{+}$cells. $\mathbf{n}-\mathbf{q}$ The histograms show the frequency of phenotypes of the CD $36^{+}$cells in the gated cells of $\mathbf{m}$. The histograms with broken lines are the fluorescence minus one negative controls for individual target molecules. The data are representative of 3 independent experiments.
$\mathrm{T}$ cells cultured with Mos isolated from naïve mice (fig. 5g).

Next, with negative selection of MACS, the $\mathrm{T}_{\text {reg }}$ cells were isolated and cocultured with CFSE-labeled CD4 ${ }^{+}$ $\mathrm{CD} 25^{-} \mathrm{T}_{\text {eff }}$ cells (labeled with CFSE) for 3 days in the presence of anti-CD3/CD28 antibodies. The results showed a marked suppressor effect of the $\mathrm{T}_{\text {reg }}$ cells on the $\mathrm{T}_{\text {eff }}$ proliferation (fig. 5h-k). The data suggest that the TSP1primed Mos have tolerogenic properties.

\section{TSP1-Primed Mos Suppress Colitis in Mice}

To test the inhibitory effect of $C$. butyricum or the TSP1-conditioned Mos in colitis, a DSS colitis mouse model was established. The results of colonic histology (fig. 5a-f), inflammatory score (fig. 5g), MPO levels (fig. 5h) of colonic tissue, the body weight and serum levels of proinflammatory cytokines (IFN- $\gamma$, TNF- $\alpha$, IL-17) showed that treatment with C. butyricum, sodium butyrate or TSP1-conditioned Mos significantly inhibited colitis (fig. 5i). In contrast, no apparent inhibitory effect on colitis was observed when the colitis mice were treated with saline or naïve Mos.

\section{Discussion}

Wen et al. [17] have reported that Lactobacillus rhamnosus GG enhances Th1 immunity, while Toomer et al. [18] propose that probiotic administration can attenuate Th2-biased cellular immunity and predisposition to food allergies. Recent work indicates that mice treated either with Bifidobacterium or Lactobacillus alone show less production of proinflammatory cytokines in the intestine and restoration of tight junction integrity [19]. In the 


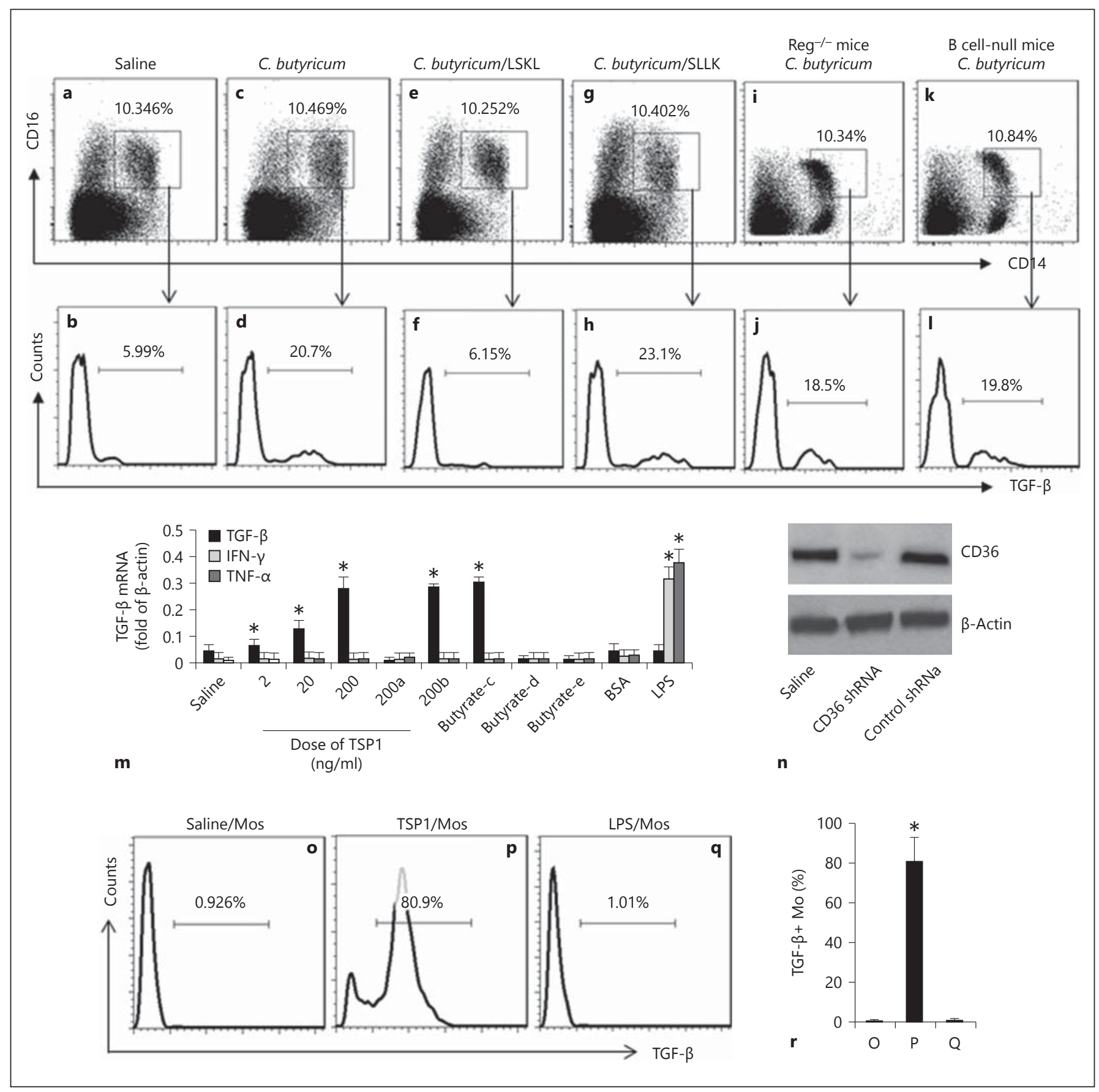

Fig. 4. C. butyricum-derived TSP 1 induces TGF- $\beta^{+}$Mos. a-j C57BL/6 mice were treated daily for 5 days as denoted above each dot plot panel. a-I The dot plots indicate the frequency of $\mathrm{CD} 14^{+}$ $\mathrm{CD}^{-} 6^{-} \mathrm{Mos}$ in the intestine; the histograms indicate the frequency of TGF- $\beta^{+}$Mos in the gated dot plots (pointed at by arrows). m Naïve Mos were generated from bone marrow cells and treated in the culture for $72 \mathrm{~h}$ as denoted on the $\mathrm{x}$-axis. The bars indicate the mRNA levels of TGF- $\beta$, IFN- $\gamma$ and TNF- $\alpha$ in the Mos. $200 \mathrm{a}=$ The ' $a$ ' indicates the Mos are CD36-deficient (by RNAi); 200b = the ' $b$ ' indicates the Mos were treated with control shRNA; butyrate-c $($ or $-\mathrm{d}$, or $-\mathrm{e})=$ the ' $c$ ' indicates that Mos were treated with sodium butyrate in the presence of IECs, the 'd' indicates that Mos were treated with sodium butyrate without the presence of IEC and the 'e' indicates that the CD36-deficient Mos were treated with sodium butyrate in the presence of IECs; BSA = bovine serum albumin; LPS = lipopolysaccharide. $\mathbf{n}$ The immune blots show the CD36 RNAi results. o-q Mos were treated in the culture as denoted above each subpanel. The histograms indicate the frequency of TGF- $\beta^{+}$Mos. $\mathbf{r}$ The bars indicate the summarized data of $\mathbf{o - q}$. The data of bars are presented as means \pm SD. ${ }^{*} p<0.01$, compared with the group in $\mathbf{0}$. The data are representative of 3 independent experiments. 


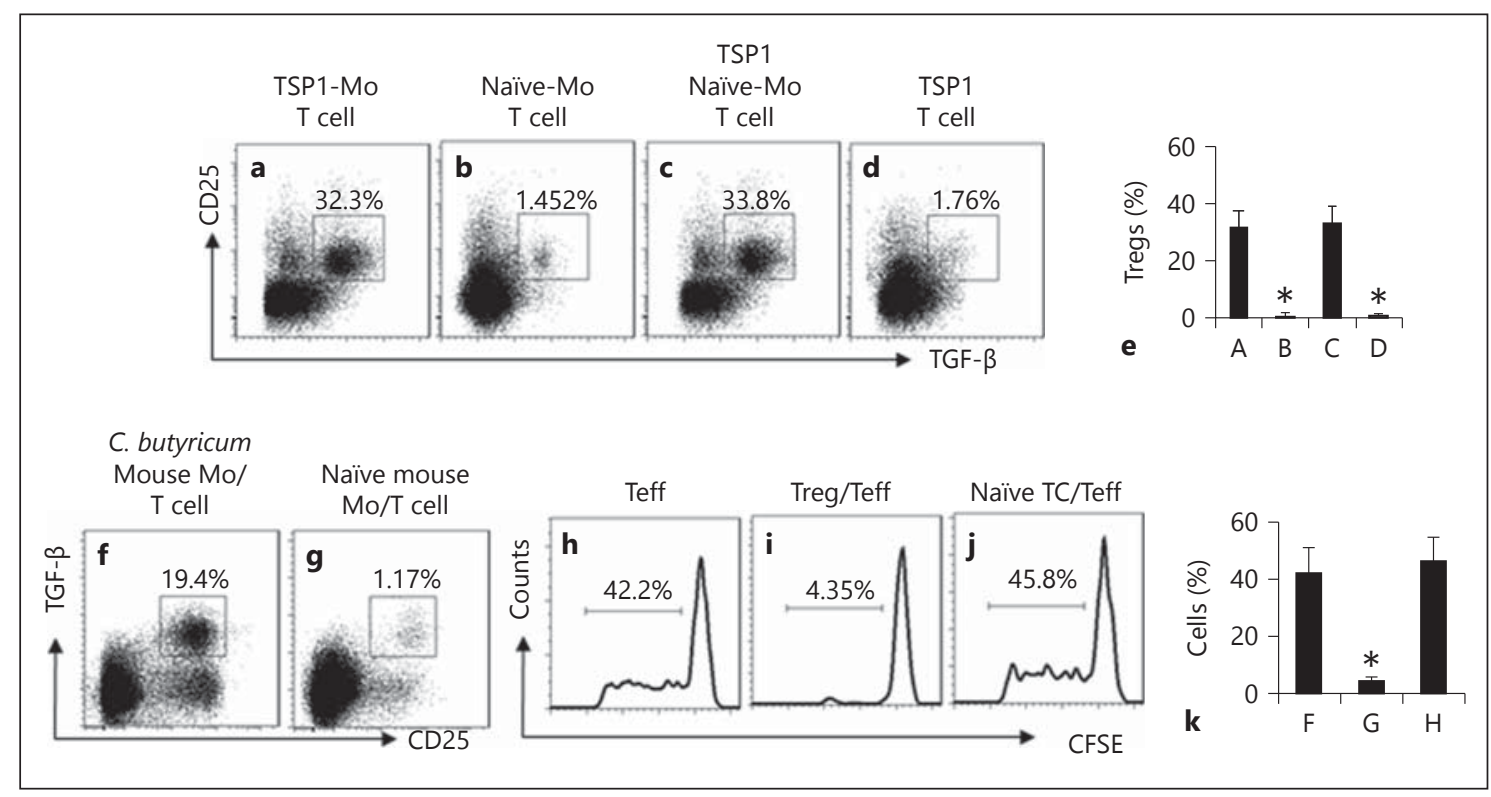

Fig. 5. TSP1-primed Mos induce $T_{\text {reg }}$ cells. a-d Mos and $T_{\text {eff }}$ cells were cultured at a ratio of $10^{4}: 10^{4}$ /well in the presence of IL-2 (20 $\mathrm{ng} / \mathrm{ml}$ ) for 6 days; the experimental design is denoted above each subpanel. The gated dot plots indicate the frequency of $\mathrm{T}_{\text {reg }}$ cells. e-g $\mathrm{T}_{\text {reg }}$ and $\mathrm{T}_{\text {eff }}$ cells (labeled with CFSE) were cultured at a ratio of $10^{4}: 10^{4}$ /well of plates coated with anti-CD3 and in the presence of CD28 $(5 \mu \mathrm{g} / \mathrm{ml})$. The histograms indicate the $\mathrm{T}_{\text {eff }}$ proliferation. The experimental design is denoted above each subpanel. The data are presented as means \pm SD as denoted in each subpanel. ${ }^{*} \mathrm{p}<$ 0.01 as compared with $\mathbf{a}(\mathbf{a}-\mathbf{d})$ or with $\mathbf{e}(\mathbf{e}-\mathbf{g})$. The data are representative of 3 independent experiments.

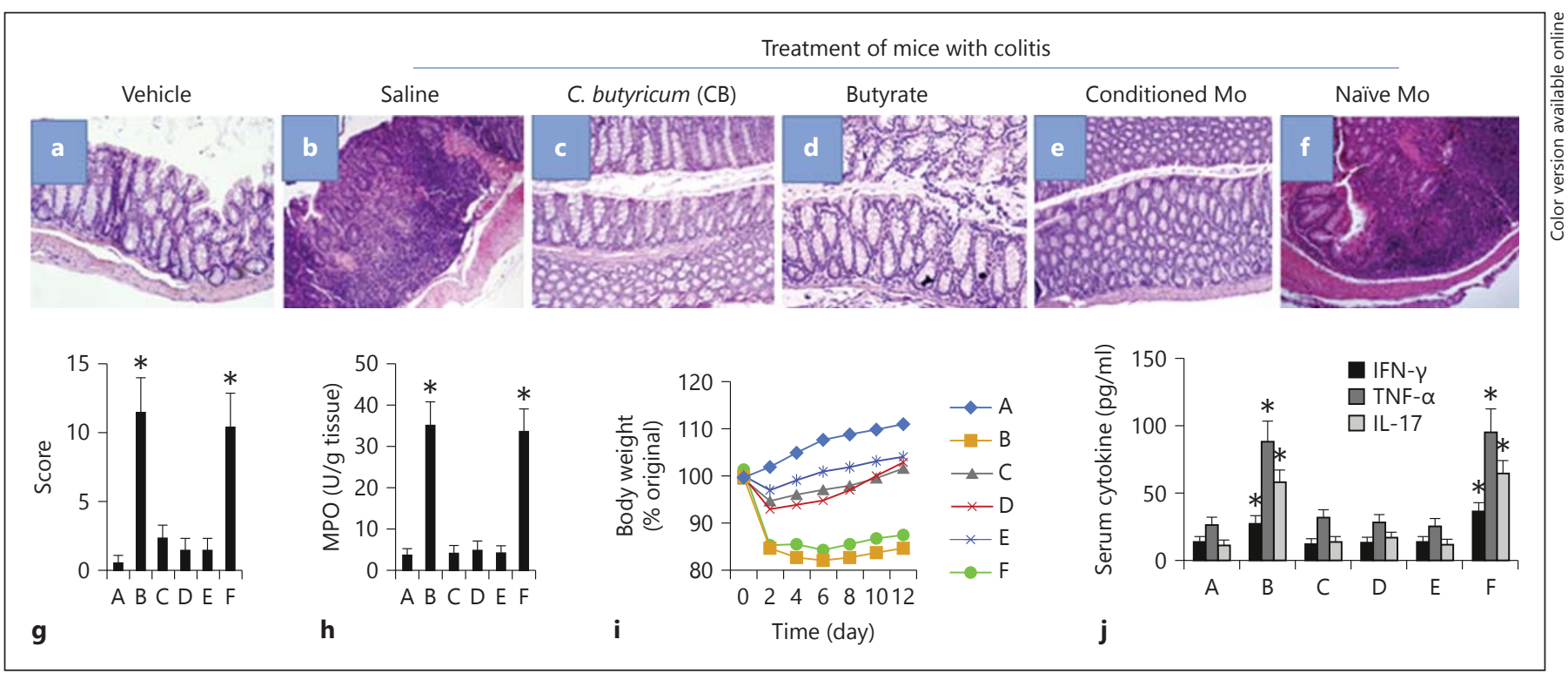

Fig. 6. TSP1-primed Mos inhibit inflammation in the intestine. a-g The representative histological images of the colon. Magnification of the images: $\times 100$. a Mice were treated with vehicle. $\mathbf{b}-\mathbf{f}$ Mice were treated with DSS; the additional treatment is denoted above each subpanel. $\mathbf{g}$, $\mathbf{h}$ The bars indicate the inflammatory scores ( $\mathbf{g})$ or the levels of MPO (h). $\mathbf{i}$ The curves indicate the body weight loss. $\mathbf{j}$ The bars indicate the serum levels of the cytokines IFN- $\gamma$, TNF- $\alpha$ and IL-17 (by ELISA). Vehicle $=50 \%$ ethanol; bu- tyrate $=$ mice were intraperitoneally injected with sodium butyrate $(1.2 \mathrm{mg} / \mathrm{kg})$; conditioned Mos = Mos were exposed to TSP 1 in the culture for $72 \mathrm{~h}$; naïve Mos $=$ Mos were cultured with medium alone. The group labels of $\mathbf{g}-\mathbf{j}$ are the same as images $\mathbf{a}-\mathbf{f}$. The data of bars are presented as means $\pm \mathrm{SD}$. ${ }^{*} \mathrm{p}<0.01$, compared with the vehicle group. Each group consists of 6 mice. Samples from individual mice were processed separately. The data are representative of 6 independent experiments. 
present study, we have revealed novel aspects of probiotics, in which the butyrate-producing C. butyricum suppresses experimental colitis via strengthening the immune tolerance in the intestine. The data suggest that $C$. butyricum or butyrate can upregulate TSP 1 expression by IECs. Thus, TSP1 may be one of the epithelial signals contributing to responses to inflammatory lesion in the gut mucosa.

Accumulating data suggest that TSP1 is often decreased or lost in human cancers [20]. The loss/downregulation of TSP1 is associated with elevated angiogenesis and malignancy [21]. However, TSP1 is also involved in inflammatory diseases, such as via activating TGF- $\beta$ to progress to chronic kidney inflammation [22], or mediates renal dysfunction [23]. Previous studies showed CD36 and TSP1 expression in the damaged mucosa of patients with IBD [24]. Our recent work has also revealed that TSP1-derived B cells promote immune tolerance in the intestinal mucosa and suppress allergic mucosal inflammation [10]. In this study, the data revealed that IECs could express detectable TSP 1 which could be markedly upregulated after activation in vitro or C. butyricum or sodium butyrate in vivo. It is well known that CD36 is a TSP1 receptor which is expressed on variable cells, including Mos [25]. In this study, we demonstrated that about $20 \%$ Mos in the mouse colon are CD36 $6^{+}$TGF- $\beta^{+}$ Mos which is independent from CD $36^{-}$TGF- $\beta^{-}$Mos, and this subset of Mos acts as $\mathrm{M}_{2}$-like macrophages ameliorating colitis in mice [26].

The composition of the human intestinal Mos pool changes considerably when there is a perturbation of homeostasis in the gut [14]. Responsive classical Mos continuously enter the steady-state intestinal mucosa as a surveillance measure has been demonstrated in previous studies [27]. Under given conditions, the Mos adopt an anti-inflammatory phenotype which is imprinted by local factors in the mucosa [27]. Our data are in line with these pioneer studies by providing further evidence that TSP1 is an important factor in conferring Mos with immunosuppressing properties and the characteristics of an antiinflammatory phenotype. Coculture of TSP1-primed Mos and $\mathrm{CD}^{+}{ }^{+} \mathrm{CD} 4^{+} \mathrm{CD} 25^{-} \mathrm{T}_{\text {eff }}$ cells revealed that such a subset of Mos could induce $\mathrm{T}_{\text {reg }}$ development in vitro. Thus, it suggests that $C$. butyricum can modulate Mo properties via epithelial cell-derived TSP1, and meanwhile, TSP1 can also convert latent TGF- $\beta$ to active TGF- $\beta$, which is able to induce the inducible $\mathrm{T}_{\text {reg }}$ cells [28].

$\mathrm{T}_{\text {reg }}$ cells play an important role in maintaining peripheral tolerance; the functional deficiency of $\mathrm{T}_{\text {reg }}$ cells is involved in the pathogenesis of various immune disorders [29]. In the gut, $\mathrm{T}_{\text {reg }}$ cells are also observed to be a primary cell population in the maintenance of immune homeostasis. Various approaches have been tried to generate $\mathrm{T}_{\text {reg }}$ cells in in vivo experiments [30]. The present data show that C. butyricum, sodium butyrate or TSP1conditioned Mos can induce an increase in the Foxp $3^{+}$ $\mathrm{T}_{\text {reg }}$ cells in sensitized mice. Such an increase could be blocked by LSKL, an inhibitor of TSP1. The data suggest that TSP1-primed Mos play a key role in inducing $\mathrm{T}_{\text {reg }}$ cells. In addition, this study further demonstrated that $\mathrm{T}_{\text {reg }}$ cells induced by TSP1-primed-Mos have an immune suppressor function in regulatory intestinal mucosal inflammation.

The present data show that Mos production of TGF- $\beta$ in response to TSP1 stimulation is of significance. Both TSP1 and TGF- $\beta$ are immunoregulatory molecules. Previous work has reported that TGF- $\beta$ is sufficient to induce the inducible $T_{\text {reg }}$ cells $[31,32]$. In line with these studies, our studies also demonstrate that TGF- $\beta^{+}$Mos can induce $\mathrm{T}_{\text {reg }}$ development in the intestine as well. Importantly, the source of TGF- $\beta$ of our study system is TSP1primed Mos. Thus, our study has revealed a novel tolerogenic cell subset, TGF- $\beta^{+}$Mos, in the body. Our findings show the plasticity of this population of Mos. Thus, it holds promise as new therapeutic target of IBD when understanding what and how the local factors determine various fates of Mos in the gut mucosa under different conditions.

In summary, the present data reveal that the treatment with $C$. butyricum could suppress experimental colitis in mice via inducing IEC-derived TSP1 and modulating Mo properties. Importantly, TSP1-primed Mos can upregulate TGF- $\beta$ levels, facilitate the generation of $\mathrm{T}_{\text {reg }}$ cells and successfully inhibit inflammation in the intestine.

\section{Acknowledgements}

This study was supported by the following grants: the Natural Science Foundation of SZU (No. 000004), the innovation of science and Technology Commission of Shenzhen Municipality (JCYJ20120613172559904), the Natural Science Foundation of China (No. 81270470; No. 81373176), and Shanghai Science and Technology Commission (12XD1404000).

\section{Disclosure Statement}

No conflict of interest to declare.
Fang/Yu/Wu/He/Li/Yan/Li/Wang/Liu/ $\mathrm{Liu} /$ Yang 


\section{References}

1 Strober W, Fuss IJ: Proinflammatory cytokines in the pathogenesis of inflammatory bowel diseases. Gastroenterology 2011;140: 1756-1767.

2 Kanai T, Mikami Y, Sujino T, Hisamatsu T, Hibi T: RORgammaT-dependent IL-17Aproducing cells in the pathogenesis of intestinal inflammation. Mucosal Immunol 2012;5: 240-247.

3 Wan YY, Flavell RA: Regulatory T cells, transforming growth factor-beta, and immune suppression. Proc Am Thorac Soc 2007;4: 271-276.

4 Komatsu N, Okamoto K, Sawa S, Nakashima T, Oh-hora M, Kodama T, Tanaka S, Bluestone JA, Takayanagi H: Pathogenic conversion of Foxp $3+$ T cells into Th17 cells in autoimmune arthritis. Nat Med 2014;20:62-68.

5 Jitschin R, Braun M, Buttner M, DettmerWilde K, Bricks J, Berger J, Eckart MJ, Krause SW, Oefner PJ, Le Blanc K, Mackensen A, Mougiakakos D: CLL-cells induce IDOhi CD14+HLA-DRlo myeloid-derived suppressor cells that inhibit T-cell responses and promote TRegs. Blood 2014;124:750-760.

6 Younos IH, Abe F, Talmadge JE: Myeloid-derived suppressor cells: their role in the pathophysiology of hematologic malignancies and potential as therapeutic targets. Leuk Lymphoma 2014;21:1-31.

7 Zhang J, Wang B, Zhang W, Wei Y, Bian Z, Zhang CY, Li L, Zen K: Protein tyrosine phosphatase $1 b$ deficiency ameliorates murine experimental colitis via the expansion of myeloid-derived suppressor cells. PloS One 2013;8:e70828.

8 Silverstein RL: The face of TSR revealed: an extracellular signaling domain is exposed. J Cell Biol 2002;159:203-206.

9 Good DJ, Polverini PJ, Rastinejad F, Le Beau MM, Lemons RS, Frazier WA, Bouck NP: A tumor suppressor-dependent inhibitor of angiogenesis is immunologically and functionally indistinguishable from a fragment of thrombospondin. Proc Natl Acad Sci U S A 1990;87:6624-6628.

10 Zhang HP, Wu Y, Liu J, Jiang J, Geng XR, Yang G, Mo L, Liu ZQ, Liu ZG, Yang PC: TSP1-producing B cells show immune regulatory property and suppress allergy-related mucosal inflammation. Sci Rep 2013;3:3345.

11 Jimenez B, Volpert OV, Crawford SE, Febbraio M, Silverstein RL, Bouck N: Signals leading to apoptosis-dependent inhibition of neovascularization by thrombospondin-1. Nat Med 2000;6:41-48.
12 Woo TD, Oka K, Takahashi M, Hojo F, Osaki T, Hanawa T, Kurata S, Yonezawa H, Kamiya S: Inhibition of the cytotoxic effect of Clostridium difficile in vitro by Clostridium butyricum Miyairi 588 strain. J Med Microbiol 2011;60:1617-1625.

13 Whelan K, Quigley EM: Probiotics in the management of irritable bowel syndrome and inflammatory bowel disease. Curr Opin Gastroenterol 2013;29:184-189.

14 Scott CL, Henri S, Guilliams M: Mononuclear phagocytes of the intestine, the skin, and the lung. Immunol Rev 2014;262:9-24.

15 Liu Z, Colpaert S, D’Haens GR, Kasran A, de Boer M, Rutgeerts P, Geboes K, Ceuppens JL: Hyperexpression of CD40 ligand (CD154) in inflammatory bowel disease and its contribution to pathogenic cytokine production. J Immunol 1999; 163:4049-4057.

16 Loewendorf AI, Nguyen TA, Yesayan MN, Kahn DA: Normal human pregnancy results in maternal immune activation in the periphery and at the uteroplacental interface. PloS One 2014;9:e96723.

17 Wen K, Tin C, Wang H, Yang X, Li G, GiriRachman E, Kocher J, Bui T, Clark-Deener S, Yuan L: Probiotic Lactobacillus rhamnosus GG enhanced Th1 cellular immunity but did not affect antibody responses in a human gut microbiota transplanted neonatal gnotobiotic pig model. PloS One 2014;9:e94504.

18 Toomer OT, Ferguson M, Pereira M, Do A, Bigley E, Gaines D, Williams K: Maternal and postnatal dietary probiotic supplementation enhances splenic regulatory $\mathrm{T}$ helper cell population and reduces ovalbumin allergen-induced hypersensitivity responses in mice. Immunobiology 2014;219:367-376.

19 Wang H, Gong J, Wang W, Long Y, Fu X, Fu Y, Qian W, Hou X: Are there any different effects of Bifidobacterium, Lactobacillus and Streptococcus on intestinal sensation, barrier function and intestinal immunity in PI-IBS mouse model? PloS One 2014, 9:e90153.

20 Sid B, Sartelet H, Bellon G, El Btaouri H, Rath G, Delorme N, Haye B, Martiny L: Thrombospondin 1: a multifunctional protein implicated in the regulation of tumor growth. Crit Rev Oncol Hematol 2004;49: 245-258.

21 Zhang J, Ito R, Oue N, Zhu X, Kitadai Y, Yoshida K, Nakayama H, Yasui W: Expression of thrombospondin-1 is correlated with microvessel density in gastric carcinoma. Virchows Arch 2003;442:563-568.
22 Zeisberg M, Tampe B, LeBleu V, Tampe D, Zeisberg EM, Kalluri R: Thrombospondin-1 deficiency causes a shift from fibroproliferative to inflammatory kidney disease and delays onset of renal failure. Am J Pathol 2014; 184:2687-2698.

23 Cui W, Maimaitiyiming H, Qi X, Norman H, Wang S: Thrombospondin 1 mediates renal dysfunction in a mouse model of high-fat diet-induced obesity. Am J Physiol Renal Physiol 2013;305:F871-F880.

24 Ortiz-Masia D, Diez I, Calatayud S, Hernandez C, Cosin-Roger J, Hinojosa J, Esplugues JV, Barrachina MD: Induction of CD36 and thrombospondin-1 in macrophages by hypoxia-inducible factor 1 and its relevance in the inflammatory process. PloS One 2012; 7:e48535.

25 Silverstein RL, Febbraio M: CD36, a scavenger receptor involved in immunity, metabolism, angiogenesis, and behavior. Sci Signal 2009;2:re3.

26 Rizzo A, Monteleone I, Fina D, Stolfi C, Caruso R, Fantini MC, Franze E, Schwendener $\mathrm{R}$, Pallone F, Monteleone G: Inhibition of colitis by IL-25 associates with induction of alternatively activated macrophages. Inflamm Bowel Dis 2012;18:449-459.

27 Bain CC, Mowat AM: Macrophages in intestinal homeostasis and inflammation. Immunol Rev 2014;260:102-117.

28 Horwitz DA, Zheng SG, Gray JD: The role of the combination of IL- 2 and TGF-beta or IL10 in the generation and function of CD4+ CD25+ and CD8+ regulatory T cell subsets. J Leukoc Biol 2003;74:471-478.

29 Sakaguchi S, Wing K, Onishi Y, Prieto-Martin P, Yamaguchi T: Regulatory T cells: how do they suppress immune responses? Int Immunol 2009;21:1105-1111.

30 Lan Q, Fan H, Quesniaux V, Ryffel B, Liu Z, Zheng SG: Induced Foxp3(+) regulatory $\mathrm{T}$ cells: a potential new weapon to treat autoimmune and inflammatory diseases? J Mol Cell Biol 2012;4:22-28.

31 Zheng SG, Gray JD, Ohtsuka K, Yamagiwa S, Horwitz DA: Generation ex vivo of TGFbeta-producing regulatory $\mathrm{T}$ cells from CD4+CD25- precursors. J Immunol 2002; 169:4183-4189.

32 Chen W, Jin W, Hardegen N, Lei KJ, Li L, Marinos N, McGrady G, Wahl SM: Conversion of peripheral CD4+CD25- naive $\mathrm{T}$ cells to CD4+CD25+ regulatory $\mathrm{T}$ cells by TGF-beta induction of transcription factor Foxp3. J Exp Med 2003;198:1875-1886. 